\title{
MANIFESTACIONES ORALES DEL SÍNDROME DE MAROTEAUX-LAMY (MUCOPOLISACARIDOSIS VI)
}

\author{
ORAL MANIFESTATIONS OF MAROTEAUX-LAMY SYNDROME \\ (MUCOPOLYSACCHARIDOSIS VI)
}

Sandra Viviana Cáceres Matta ${ }^{*}$ (1) scacerem09@gmail.com

Luis Eduardo Carmona Arango' ${ }^{(1)}$ lcarmonaa@unicartagena.edu.co

Artículo recibido: 12/11/2020

Arbitrado por pares

Artículo aceptado: 12/03/2021

Artículo publicado: 25/03/2021

*Autor corresponsal:

Sandra Viviana Cáceres Matta

scacerem09@gmail.com

\section{RESUMEN}

La mucopolisacaridosis tipo VI, también conocida como síndrome de Maroteaux-Lamy, es un trastorno lisosómico autosómico recesivo, causado por la deficiencia de la enzima arilsulfatasa $\mathrm{B}$, lo que conduce a la acumulación de dermatán sulfato en los tejidos y su excreción urinaria. La deposición de mucopolisacáridos genera un trastorno progresivo que afecta a múltiples órganos y que, a menudo, resulta en la muerte a temprana edad. Esta enfermedad tiene varias manifestaciones orales, entre las que destacan las complicaciones dentales, que pueden ser graves e incluir folículos similares a quistes dentígeros, maloclusiones, defectos condilares e hiperplasia gingival, además de características clínicas como cuello corto, opacidad corneal, macroglosia y agrandamiento del cráneo, dimensión anteroposterior larga y mano en garra. Se presenta el caso de un paciente de 14 meses de edad que acudió a consulta de odontopediatría por episodios de fiebre, bajo peso e hiperplasia gingival severa. El examen físico evidenció facies tosca, cuello corto, pectus excavatus, manos con disminución en agarre y retardo en el neurodesarrollo. El examen intraoral halló retardo de la erupción dental, hiperplasia gingival generalizada y paladar con poco crecimiento transversal. El examen radiográfico detectó órganos dentarios incluidos y mala posición en el sector anterior, molares superiores dentro del seno maxilar y caninos inferiores rotados. El paciente fue remitido a medicina para exámenes bioquímicos y genéticos para definir el diagnóstico. La bioquímica reveló MPS tipo VI, lo que fue confirmado mediante prueba molecular. Las manifestaciones clínicas en este caso corresponden a la forma clínica de progresión rápida reportada en estos pacientes: talla baja, malformaciones esqueléticas y alteraciones a nivel oral. Los niños con MPS VI grave comienzan temprano y progresan rápidamente, las radiografías óseas y la medición de GAG en orina son útiles para el diagnóstico con actividad de la enzima ARSB y genética. Es necesario fortalecer el conocimiento en odontología y la población en general sobre las características clínicas de mucopolisacáridos tipo VI para tener un diagnóstico temprano y un mejor manejo de patologías en estos pacientes.

Palabras clave: mucopolisacáridos, arilsulfatasa, dermatán sulfato, hiperplasia gingival

\section{ABSTRACT}

Mucopolysaccharidosis type VI, also known as Maroteaux-Lamy syndrome, is an autosomal recessive lysosomal disorder, due to the deficiency of the enzyme arylsulfatase B that leads to the accumulation of dermatan sulfate in the tissues and its urinary excretion. Mucopolysaccharide deposition leads to a progressive disorder affecting multiple organs that often results in death at a young age. This disease has several oral manifestations, among which dental complications can be serious and include follicles similar to dentigerous cysts, malocclusions, condylar defects and gingival hyperplasia, in addition to a short neck, corneal opacity, macroglossia, skull enlargement, anteroposterior dimension long, claw hand is some of the clinical features. A case of a 14-monthold patient is presented, who attended a pediatric dentistry consultation for episodes of fever, low
\end{abstract}

Citar como: Cáceres S, Carmona L. Manifestaciones orales del síndrome de Maroteaux-Lamy (mucopolisacaridosis VI). Rev Cient Odontol (Lima). 2021; 9 (1): e051.

DOI: 10.21142/2523-2754-0901-2021-051

Grupo de Investigación PROMOUC, Programa de Odontología, Facultad Ciencias de la Salud de la Universidad del Sinú Seccional Cartagena. Cartagena,
Colombia.

2 Grupo de Investigación PROMOUC, Posgrado de Odontopediatría y Ortopedia Maxilar, Facultad de Odontología de la Universidad de Cartagena. Cartagena, Colombia. 
weight, severe gingival hyperplasia. Physical examination revealed coarse facies, short neck, pectus excavatus, hands with decreased grip, and neurodevelopmental delay. On intraoral examination, dental eruption delayed, generalized gingival hyperplasia, palate with little transverse growth. On radiographic examination, dental organs included and poor position in the anterior sector, upper molars within the maxillary sinus, rotated lower canines. He is referred to medicine for biochemical tests and genetics for diagnosis. Detailed biochemistry MPS type VI, confirmed by molecular testing. The clinical manifestations in this case correspond to the clinical form of rapid progression reported in these patients. They report: short stature, skeletal malformations and alterations at the oral level. Children with severe MPS VI start early and progress rapidly, bone radiographs and urine GAG measurement are helpful for diagnosis with genetic and ARSB enzyme activity. It is necessary to strengthen the knowledge in dentistry and the general population about the clinical characteristics of type VI mucopolysaccharides in order to have an early diagnosis and management of pathologies in these patients.

Keywords: mucopolysaccharides, arylsulfatase, dermatan sulfate, gingival hiperplasia

\section{INTRODUCCIÓN}

Las mucopolisacaridosis son un grupo de trastornos genéticos que implican alteraciones en el metabolismo de los mucopolisacáridos. Las manifestaciones clínicas son resultado de la acumulación de mucopolisacáridos (glicosaminoglicanos) en varios órganos. El depósito de mucopolisacáridos conduce a retraso en el neurodesarrollo desde los primeros meses de vida. Las mucopolisacaridosis son autosómicas recesivas, con excepción del síndrome de Hunter, que se hereda como un rasgo recesivo ligado al cromosoma $\mathrm{X}(1,2,3)$.

El síndrome de Maroteaux-Lamy (mucopolisacaridosis tipo VI), descrito por primera vez en $1965\left({ }^{4}\right)$, se debe a una deficiencia de la enzima arilsulfatasa B (N-acetilgalactosamina4-sulfatasa), que genera la acumulación del dermatán sulfato dentro de los lisosomas $\left.{ }^{5}\right)$. Normalmente, los pacientes con mucopolisacaridosis tipo VI se diagnostican en los primeros años de vida y las afecciones más las fallas multisistémicas, por lo general, ocasionan la muerte temprana.

Los pacientes que sufren este síndrome tienen características similares a los que padecen otras mucopolisacaridosis, pero se distinguen por la inclusión de metacromáticas en los leucocitos y una actividad deficiente de la arilsulfatasa $\mathrm{B}\left({ }^{6}\right)$. Los rasgos característicos de la enfermedad incluyen retraso en el crecimiento, hernias, facies tosca, cuello corto, anomalías en la columna, mano en garra y hepatoesplenomegalia (resultado del depósito de mucopolisacáridos en varios órganos). En la mayoría de los casos, la muerte se debe a la infección del tracto respiratorio o a una enfermedad cardíaca.

Las características creneofaciales en este síndrome son similares a las del síndrome de Hurler (MPS tipo I). La cabeza presenta un aumento de tamaño, con frente prominente, a menudo la zona supraorbital; crestas y protuberancias temporales marcadas. La rinitis con rinorrea y la opacidad corneal son comunes en estos pacientes. A nivel de cavidad oral, se observa incompatibilidad labial y macroglosia. Algunas investigaciones han reportado órganos dentarios en forme de clavija, anormales, con anomalías de número y forma, al igual que defectos en la calcificación dental como hipoplasias; normalmente, existe una mordida abierta anterior asociada a la macroglosia $\left({ }^{7,8}\right)$.

El objetivo de este artículo es describir las características clínicas odontológicas específicas de un paciente portador del síndrome de Maroteaux-Lamy (mucopolisacaridosis tipo VI).

\section{REPORTE DE CASO}

Una niña de 14 meses de edad, de procedencia rural, del departamento de Bolívar (Colombia), con síndrome 
de Maroteaux-Lamy (mucopolisacaridosis tipo VI), fue remitida al Posgrado de Odontopediatría y Ortopedia Maxilar de la Universidad de Cartagena (Colombia) para el diagnóstico odontológico como complemento del reporte médico, el cual señalaba hepatoesplenomegalia leve. La excreción urinaria de mucopolisacaridosis media, con la prueba de azul de dimetilmetileno, desde el área de bioquímica, arrojó $54,7 \mathrm{mg} / \mathrm{mmol}$ de creatinina (valores normales: $<12,2 \mathrm{mg} / \mathrm{mmol}$ creatinina). La electroforesis bidimensional de mucopolisacaridosis en muestra de orina mostró un gran aumento de dermatán sulfato, lo que sugiere mucopolisacaridosis tipo VI. La deficiencia enzimática de la arilsulfatasa B fue identificada por ensayo de enzima ASB $\left({ }^{9}\right)$ de los fibroblastos cultivados de la paciente, la actividad de ABS fue 120 y el rango control de 300 a $900 \mathrm{nmol} / \mathrm{h} / \mathrm{mg}$.

De igual manera, la paciente en la historia clínica médica reportaba insuficiencia cardiaca y respiratoria. Los exámenes radiográficos evidenciaron rasgos típicos como disostosis múltiple. El consentimiento informado escrito y verbal se obtuvo antes de la documentación del caso; asimismo, se le explicó a la madre de la paciente cada uno de los procedimientos odontológicos por desarrollar. Los hallazgos extraorales e intraorales de la paciente también

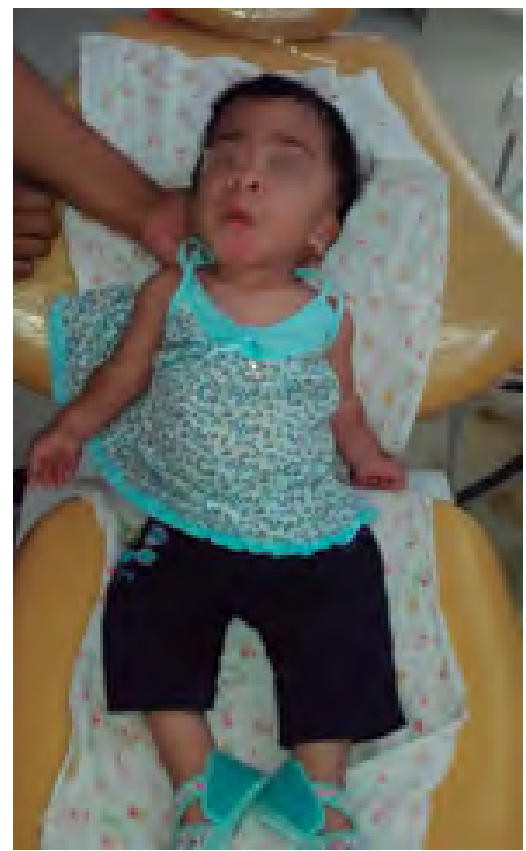

Figura I. Esta fotografía muestra la facies característica del síndrome de Maroteaux-Lamy. fueron característicos del síndrome Maroteaux-Lamy, pues presentaba retraso en el desarrollo psicomotor, cuello corto, mano en forma de garra (figura 1), mordida abierta anterior asociada con hiperplasia gingival y leve macroglosia, agrandamiento leve del cráneo y una dimensión anteroposterior aumentada, puente nasal aplanado e hipertelorismo, cejas pobladas, las pestañas estaban presentes (figuras $2 \mathrm{~A}$ y $2 \mathrm{~B}$ ).

El examen clínico intraoral reveló hiperplasia gingival, retraso en la erupción dental de órganos dentarios anteriores, agradamiento de procesos alveolares, paladar profundo, estrecho en sentido sagital y trasversal, macroglosia (figuras $3 \mathrm{~A}$ y $3 \mathrm{~B}$ ).

Se tomó radiografía panorámica en circunstancias difíciles (la paciente no cooperaba y estaba ansiosa), el estudio radiográfico reveló dientes no erupcionados

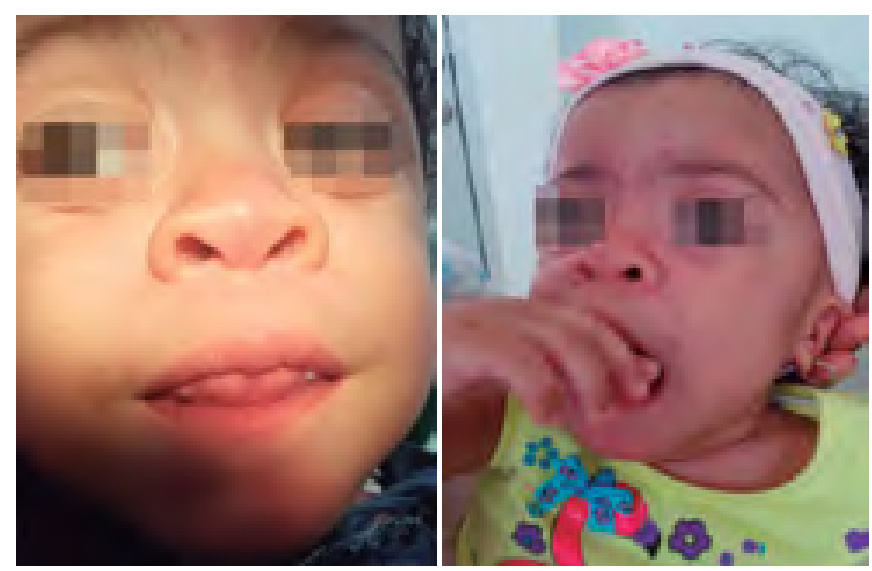

Figura $2 \mathrm{~A}$ у 2 B. Cabeza grande, cuello corto, incompetencia labial por hiperplasia gingival y leve lengua agrandada, agrandamiento del cráneo y una dimensión anteroposterior larga son características típicas del síndrome de Maroteaux-Lamy.
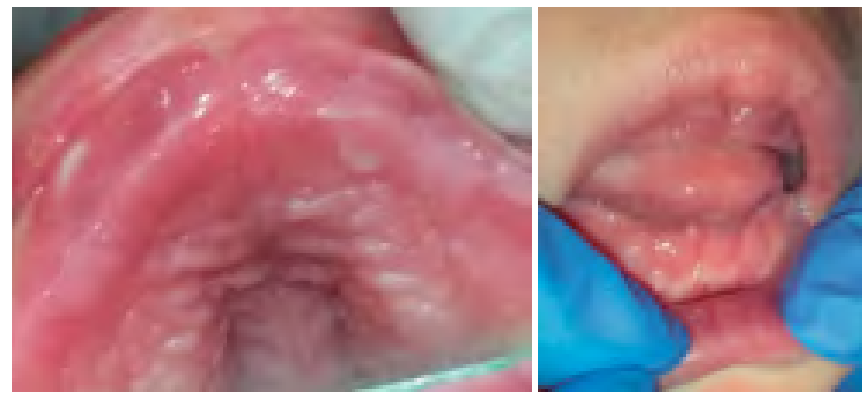

Figura 3 A y 3 B. Hiperplasia gingival generalizada en maxilar superior e inferior, retraso en la erupción de los dientes anteriores, paladar profundo con poco desarrollo en el crecimiento del plano medio sagital, macroglosia. 


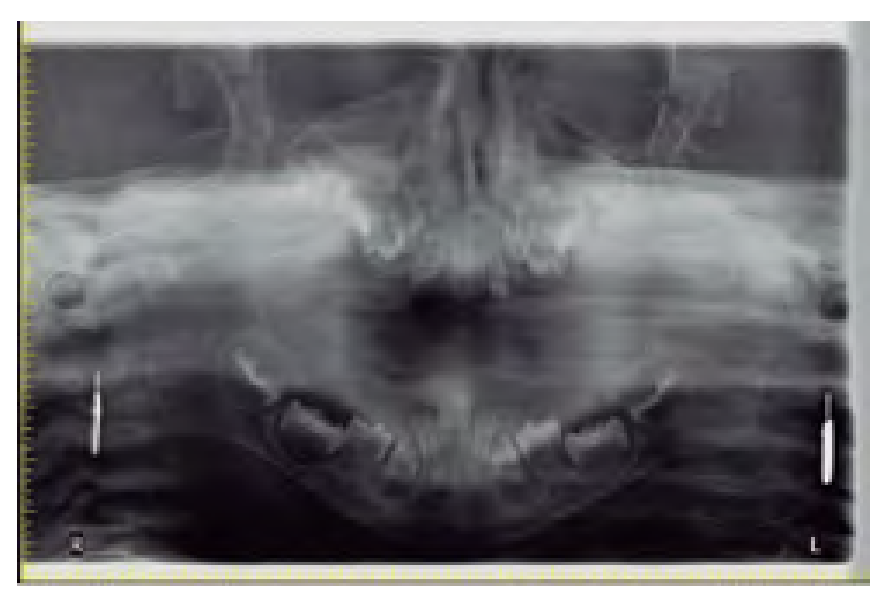

Figura 4. La radiografía panorámica muestra los dientes no erupcionados y varias radiolucencias pericoronales.

con radiolucencias pericoronales que se asemejaban a quistes dentígeros (figura 4). El árbol genealógico de 3 generaciones de la familia reveló consanguinidad (figura 5).

\section{DISCUSIÓN}

Este raro síndrome es una enfermedad autosómica recesiva causada por mutaciones en la enzima arilsulfatasa B y el gen ARSB, necesario para la degradación de dermatán sulfato. Un defecto en el gen da como resultado la acumulación de mucopolisacáridos no degradados o parcialmente degradados, que luego interfieren con la función de las células afectadas, lo que da como resultado labios grandes, gruesos, hiperplasias gingivales y opacidades corneales $\left({ }^{10,11}\right)$. Su prevalencia es baja y varía de 1:100 000 nacidos a 1:1300 000, en diversas poblaciones $\left({ }^{12}\right)$. La acumulación progresiva de mucopolisacáridos en tejido blando causa complicaciones como insuficiencia cardiaca y bronconeumonía, que ocasionan la muerte de estos pacientes en los primeros años de vida $\left({ }^{7}\right)$.

Diversas investigaciones describen a los órganos dentarios de estos pacientes como de tamaño pequeño y espaciados, de igual manera, y se han reportado anomalías en número y forma con alteraciones en la calcificación, mordida abierta anterior asociada con la hiperplasia gingival y macroglosia $\left({ }^{13}\right)$. Los rasgos faciales característicos de Maroteaux-Lamy son un aumento de tamaño de la

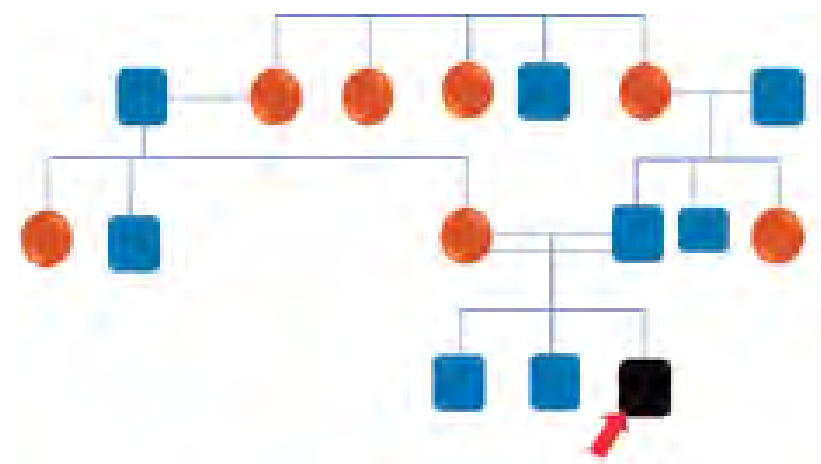

Figura 5. El familiograma del árbol genealógico de tres generaciones de la familia reveló consanguinidad.

cabeza en la zona anteroposterior y cuello corto (figura 1). Aunque haya erupción tardía de incisivos, se evidencia hipocalcificación con alteración de forma. Kantaputra reportó que los pacientes con síndrome de MaroteauxLamy tienen muy poca caries dental. Las hiperplasias gingivales y la hipertrofia de la cresta alveolar maxilar $\left(^{5}\right)$ se reportan como las principales manifestaciones orales del síndrome, tal como se evidencia en las figuras $3 \mathrm{~A}$ y 3B.

Además de estos hallazgos orofaciales, el síndrome también describe áreas radiolúcidas que se asemejan a folículos similares a un quiste dentígero $\left({ }^{13}\right)$. Se detectaron áreas radiolúcidas similares en nuestra paciente (figura 4); los márgenes de la radiolucencias suelen ser suaves y claramente definidos. Estas radiotransparencias son causadas por la acumulación de mucopolisacáridos en tejidos $\left({ }^{1,2}\right)$. El odontopediatra tiene un papel relevante en el cuidado de la salud bucal de estos pacientes.

El estudio de las enfermedades de almacenamiento de carbohidratos es sumamente importante para comprender cuáles son las consecuencias que puede tener un defecto genético que se expresa en el mal funcionamiento de un organelo, ya que debido a su poca frecuencia en la población no se tiene presente su comportamiento y resulta difícil crear un tratamiento efectivo. Se debe tener en cuenta que la calidad de vida de las personas que las padecen es muy baja y por ello es necesario ampliar los estudios, pues por lo general solo reciben cuidados de naturaleza paliativa. La aplicación de métodos de 
vanguardia en diversos campos para unificarlos podrían hacer realidad un tratamiento efectivo, enfocado en la edición genética, de forma que se pueda combatir de raíz las enfermedades de carácter autosómico recesivo, como la mucopolisacaridosis, y de esta forma tener certeza de obtener un resultado con una efectividad muy alta y tal vez definitiva $\left({ }^{3,4}\right)$.

De acuerdo con lo anterior, las enfermedades lisosomales en conjunto tienen una incidencia de 1:7700 nacidos vivos, cantidad a considerar por el gremio odontológico que, como en el presente caso, pueden ser los llamados a realizar un diagnóstico temprano, basado en la cantidad de manifestaciones orales presentes, que convierten al profesional de la odontología en una remisión obligada. Al revisar las manifestaciones clínicas generales de las enfermedades lisosomales $\left({ }^{13}\right)$, solo la macroglosia es reportada como un hallazgo frecuente, patología presente en muchos síndromes. Se hace necesario que el odontólogo y, específicamente, el odontopediatra conozca casos clínicos donde se presenten otros hallazgos que le permitan relacionar las manifestaciones generales con las intraorales, y brindar así una orientación y un manejo efectivo de estas patologías huérfanas.

\section{CONCLUSIONES}

$\mathrm{El}$ pronóstico de estos pacientes varía en función de la edad de aparición, de la velocidad de progresión de la enfermedad, de la edad de inicio del tratamiento del suplemento enzimático y del manejo interdisciplinario. Esta patología se caracteriza por ser crónica y progresiva, por lo que el inicio temprano del tratamiento permite mayores logros terapéuticos y mejor calidad de vida para los pacientes y sus familias. Por ello, es necesario que exista mayor conocimiento entre la comunidad médico-odontológica y la población en general sobre las características clínicas de la mucopolisacaridosis tipo VI, para lograr un diagnóstico temprano y un mejor manejo de las patologías que presentan estos pacientes portadores del síndrome.

Contribución de los autores: Sandra Viviana Cáceres Matta realizó los procedimientos clínicos del caso y redactó el manuscrito. Luis Eduardo Carmona Arango asesoró los procedimientos clínicos y aprobó y editó la versión final del manuscrito.

Potenciales conflictos de interés: Los autores declaran que no existe conflicto de intereses.

Fuente de financiamiento: Autofinanciado.

Agradecimientos: Al Dr. Ángel Castro Dager, médico pediatra, y a los padres de la paciente, quienes accedieron a la publicación del caso clínico en memoria de su hija, que falleció a la edad de dos años. 


\section{REFERENCIAS BIBLIOGRÁFICAS}

1. Nelson J, Crowhurst J, Carey B, Greed L. Incidence of the mucopolysaccharidoses in Western Australia. Am J Med Genet A. 2003; 123A (3): 310-3. doi: 10.1002/ajmg.a.20314.

2. Lin HY, Lin SP, Chuang CK, Niu DM, Chen MR, Tsai FJ, Chao MC, Chiu PC, Lin SJ, Tsai LP, Hwu WL, Lin JL. Incidence of the mucopolysaccharidoses in Taiwan, 1984-2004. Am J Med Genet A. 2009; 149A (5): 960-4. doi: 10.1002/ajmg.a.32781.

3. Baehner F, Schmiedeskamp C, Krummenauer F, Miebach E, Bajbouj M, Whybra C, Kohlschütter A, Kampmann C, Beck M. Cumulative incidence rates of the mucopolysaccharidoses in Germany. J Inherit Metab Dis. 2005; 28 (6): 1011-7. doi: 10.1007/s10545-005-0112-z.

4. Cavaleiro RM, Pinheiro Md, Pinheiro LR, Tuji FM, Feio Pdo S, de Souza IC, Feio RH, de Almeida SC, Schwartz IV, Giugliani R, Pinheiro JJ, Santana-da-Silva LC. Dentomaxillofacial manifestations of mucopolysaccharidosis VI: clinical and imaging findings from two cases, with an emphasis on the temporomandibular joint. Oral Surg Oral Med Oral Pathol Oral Radiol. 2013; 116 (2): e141-8. doi: 10.1016/j.oooo.2013.04.021.

5. Kantaputra PN, Kayserili H, Güven Y, Kantaputra W, Balci MC, Tanpaiboon P, Uttarilli A, Dalal A. Oral manifestations of 17 patients affected with mucopolysaccharidosis type VI. J Inherit Metab Dis. 2014; 37 (2): 263-8. doi: 10.1007/s10545-013-96458. Epub 2013 Aug 22.

6. Wadenya RO, Stout AM, Gupta A, Monge J. Hurler syndrome: a case report of a 5-year follow-up of dental findings after bone marrow transplantation. Spec Care Dentist. 2010; 30 (1): 14-7. doi: 10.1111/j.1754-4505.2009.00115.x.

7. Lachman RS, Burton BK, Clarke LA, Hoffinger S, Ikegawa S, Jin DK, Kano H, Kim OH, Lampe C, Mendelsohn NJ, Shediac R, Tanpaiboon P, White KK. Mucopolysaccharidosis IVA (Morquio A syndrome) and VI (Maroteaux-Lamy syndrome): under-recognized and challenging to diagnose. Skeletal Radiol. 2014; 43 (3): 359-69. doi: 10.1007/s00256-013-1797-y.
8. McGovern E, Owens L, Nunn J, Bolas A, Meara AO, Fleming P. Oral features and dental health in Hurler Syndrome following hematopoietic stem cell transplantation. Int J Paediatr Dent. 2010; 20 (5): 322-9. doi: 10.1111/j.1365-263X.2010.01055.x.

9. Ferreira Dos Reis M, Pinheiro LR, Pinheiro MDGR, de Almeida HA, Feio PDSQ, de Almeida SC, de Souza ICN, Giugliani R, Schwartz IVD, Cavaleiro RMDS, Pinheiro JJV, da Silva LCS. Mucopolysaccharidosis VI and effects on growth of the apical bases: a case report. Spec Care Dentist. 2018; 38 (3): 176-84. doi: 10.1111/scd.12290.

10. Vieira T, Schwartz I, Muñoz V, Pinto L, Steiner C, Ribeiro M, Boy R, Ferraz V, de Paula A, Kim C, Acosta A, Giugliani R. Mucopolysaccharidoses in Brazil: what happens from birth to biochemical diagnosis? Am J Med Genet A. 2008; 146A (13): 1741-7. doi: 10.1002/ajmg.a.32320.

11. Ponciano S, Areias C, Leão-Teles E, Sampaio-Maia B. Hyposalivation, acidic saliva, decayed teeth and oral yeast prevalence in children with mucopolysaccharidosis. Medical Express 2015; 2: M150502. DOI: 10.5935/ MedicalExpress.2015.05.02

12. James A, Hendriksz CJ, Addison O. The oral health needs of children, adolescents and young adults affected by a mucopolysaccharide disorder. JIMD Rep. 2012; 2: 51-8. doi: 10.1007/8904_2011_46.

13. Antunes LA, Nogueira AP, Castro GF, Ribeiro MG, de Souza IP. Dental findings and oral health status in patients with mucopolysaccharidosis: a case series. Acta Odontol Scand. 2013; 71 (1): 157-67. doi: 10.3109/00016357.2011.654255. 